\title{
Finite-dimensional perturbations of linear operators and some applications to boundary integral equations
}

\author{
S. E. Mikhailov \\ Wessex Institute of Technology, Ashurst Lodge, Ashurst, Southampton, SO40 7AA, UK \\ (mik@wessex.ac.uk).
}

\begin{abstract}
Finite-dimensional perturbing operators are constructed using some incomplete information about eigen-solutions of an original and/or adjoint generalized Fredholm operator equation (with zero index). Adding such perturbing operator to the original one reduces the eigen-space dimension and can, particularly, lead to an unconditionally and uniquely solvable perturbed equation. For the second kind Fredholm operators, the perturbing operators are analysed such that the spectrum points for an original and the perturbed operator coincide except a spectrum point considered, which can be removed for the perturbed operator. A relation between resolvents of original and perturbed operators is obtained. Effective procedures are described for calculation of the undetermined constants in the right-hand side of an operator equation for the case when these constants must be chosen to satisfy the solvability conditions not written explicitly. Implementation of the methods is illustrated on a boundary integral equation of elasticity.
\end{abstract}

Keywords: Linear operators; Perturbation; Resolvent; Spectrum; Eigenspace; Boundary integral equations; Elasticity

\section{Introduction}

Boundary integral equations (BIE) for boundary value problems of mathematical physics are often not unconditionally and uniquely solvable. As a consequence, the linear algebraic equation system, which is a discrete analogue of the corresponding boundary integral equation, is ill-conditioned. To avoid this difficulty, it is possible to add a finite-dimensional operator to an original boundary integral equation and to obtain an unconditionally and uniquely solvable perturbed BIE. This equation provides a solution of the original BIE if its right-hand side satisfies the original solvability condition. As heuristic, this approach was used by D.I.Sherman (see [1]) for some integral equations of two-dimensional elasticity. 
Let us consider a direct BIE of three-dimensional isotropic homogeneous elasticity for illustration. We suppose summation in repeating indices from 1 to 3 unless another range is explicitly given. It is well known (see, e.g., [2]) that the boundary value problem of elasticity in a domain $D$ with prescribed tractions $t_{j}$ at the boundary $S$ and volume forces $f_{j}$ in the domain $D$ can be reduced by the direct approach to the following BIE (for $\lambda=-1$ )

$$
\begin{gathered}
u_{i}(\xi)-\lambda\left[\underline{W}_{i j} u_{j}\right](\xi)=\Phi_{i}(\xi),\left[\underline{W}_{i j} u_{j}\right](\xi):=2 \int_{S} T_{i j}(\xi, \eta) u_{j}(\eta) d S(\eta) \\
\Phi_{i}(\xi):=2 \int_{S} U_{i j}(\xi, \eta) t_{j}(\eta) d S(\eta)+2 \int_{D} U_{i j}(\xi, \eta) f_{j}(\eta) d D(\eta) .
\end{gathered}
$$

The kernel $U_{i j}(\xi, \eta)$ is the Kelvin fundamental solution, $T_{i j}(\xi, \eta)$ is its traction vector, and $-\frac{1}{2} W u$ is the elastic double layer potential. It is known (see, e.g., $[3,4,5]$ ) that, for a bounded domain $D$ there are no singular points of the resolvent of $\underline{W}$ in the closed circle $|\lambda| \leq 1$ except the point $\lambda=-1$ being a simple pole of the resolvent, $\operatorname{dim} \operatorname{ker}(I+\underline{W})=6$, the eigen-solutions of homogeneous BIE (1) at $\lambda=-1$ are given by the six rigid body motions

$$
\stackrel{\circ}{u}_{i}^{(m)}(\xi)=\delta_{i m}, \quad \stackrel{\circ}{u}_{i}^{(3+m)}(\xi)=\epsilon_{i j m} \xi_{j}, \quad i, j, m=1, \ldots, 3,
$$

where $\epsilon_{i j m}$ is the Levi-Civita permutation tensor. Inhomogeneous BIE (1) is solvable only if its right hand side satisfies solvability conditions

$$
\int_{S} \Phi_{i}(\xi) \stackrel{\circ}{x}_{i}^{*(m)}(\xi) d S=0, \quad m=1, \ldots, 6,
$$

where the functions $\stackrel{\circ}{x}_{i}^{*}(m)(\xi)$ are generally not known.

For mechanically meaningful problems with zero total force and moments applied to the domain $D$ and its boundary, conditions (3) are always fulfilled. However these conditions may be violated in numerical solving because of discretization and round-off errors. To avoid this difficulty, it is usual in the numerical practice to fix displacements at several points, that is, to replace the given problem with prescribed tractions by a mixed problem. This means that the non-zero total force and moment, arising due to discretization errors, are transferred to these points and may cause an increased error there. Moreover, such replacement changes the BIE (1) spectral properties and can prevent application of iterative methods for its solution. Another possibility to eliminate the instability from discretization and round-off errors and improve the BIE spectral properties, is to perturb (1) by a finite-dimensional operator and to solve the perturbed equation. The second possibility will be described at the end of the paper.

For operator equations of the first kind in Banach spaces, the general principle of the choice of finite-dimensional perturbing operators can be based on the generalized Schmidt lemma, which was proved for a particular case in [6] (see also [7]). If a second-kind equation is considered, there is a sense to chose a perturbing operator so that spectrum points after the perturbation are not changed excepting one spectrum point at which the equation is to be solved. The perturbed operator spectrum is determined by the original operator spectrum and the Weinstein-Aronszajn determinant (see [8]). This determinant can not be always calculated. In [9], $\S 3$, such perturbed operators was studied for operators in 
Banach spaces using the knowledge of all eigen-solutions of the original or of the adjoint equation.

A development of the study of finite-dimensional perturbed operators is presented here. Using these results, one can remove a spectrum point of an operator equation and, if it is necessary, construct a choice procedure for unknown constants in the right-hand side of the equation. By this procedure, it is possible to make the original equation solvable.

Let $B_{1}$ and $B_{2}$ be Banach spaces, $B_{1}^{*}$ and $B_{2}^{*}$ be adjoined (dual) spaces of bounded linear functionals defined on $B_{1}$ and $B_{2}$ respectively. Let $\underline{A}$ be a linear bounded operator acting from $B_{1}$ to $B_{2}, \underline{A}: B_{1} \rightarrow B_{2}$.

Consider an operator equation

$$
\underline{A} x=y,
$$

where $x \in B_{1}, y \in B_{2}$. An adjoined equation to (4) is an equation

$$
\underline{A}^{*} x^{*}=y^{*}
$$

where $\underline{A}^{*}: B_{2}^{*} \rightarrow B_{1}^{*}, x^{*} \in B_{2}^{*}, y^{*} \in B_{1}^{*}$. Equations (4) of the form

$$
\left(\underline{I}-\lambda \underline{A}_{0}\right) x=y
$$

(second-kind operator equations) are also studied in this paper. Here $\underline{A}_{0}: B \rightarrow B$; $\underline{I}$ is the identity operator; $x, y \in B ; \lambda$ is a complex parameter. The equation

$$
\left(\underline{I}-\lambda \underline{A}_{0}^{*}\right) x^{*}=y^{*}
$$

is an adjoint equation to (6), where $\underline{A}_{0}^{*}: B^{*} \rightarrow B^{*} ; x^{*}, y^{*} \in B^{*}$.

If elements $x_{i} \in E(i=1, \ldots, n)$ are a basis of an $n$-dimensional manifold $E$, we write $E=\operatorname{span}\left\{x_{i}\right\}_{i=1}^{n}$.

Hypothesis 1 Suppose hereafter, that $\underline{A}$ is defined in the whole space $B_{1}$ and its range $\mathcal{R}(\underline{A})$ belongs to $B_{2}$ and is closed. Suppose also that $\underline{A}: B_{1} \rightarrow B_{2}$ is a Fredholm (with zero index) operator, that is, $\operatorname{dim} \operatorname{ker} \underline{A}=\operatorname{dim} \operatorname{ker} \underline{A}^{*}=n<\infty$, where $\operatorname{ker} \underline{A}=\operatorname{span}\left\{\stackrel{\circ}{x}_{i}\right\}_{i=1}^{n}$ $\subset B_{1}, \operatorname{ker} \underline{A}^{*}=\operatorname{span}\left\{\stackrel{\circ}{x}_{i}^{*}\right\}_{i=1}^{n} \subset B_{2}^{*}$ are eigen-spaces (for the eigen-value zero).

It is well known (see, e.g., $[7,8]$ ) that under Hypothesis 1, equation (4) is solvable for an element $y \in B_{2}$ iff

$$
\stackrel{\circ}{x}_{i}^{*}(y)=0 \quad(i=1, \ldots, n)
$$

for the functionals $\stackrel{\circ}{x}_{i}^{*}: \operatorname{ker} \underline{A}^{*}=\operatorname{span}\left\{\stackrel{\circ}{x}_{i}^{*}\right\}_{i=1}^{n}$.

\section{Finite-dimensional perturbations for Fredholm operator equations of the first kind}

Consider equation (4) and the equation perturbed by a finite-dimensional operator

$$
\left(\underline{A}-\underline{A}_{1}\right) x=y, \quad \underline{A}_{1} x:=\sum_{i=1}^{k} \psi_{i} \varphi_{i}(x),
$$


where $\psi_{i}$ belong to $B_{2}$ and functionals $\varphi_{i}$ belong to $B_{1}^{*}$. The equation

$$
\left(\underline{A}^{*}-\underline{A}_{1}^{*}\right) x^{*}=y^{*}, \quad \underline{A_{1}^{*} x^{*}}:=\sum_{i=1}^{k} x^{*}\left(\psi_{i}\right) \varphi_{i}
$$

is an adjoint equation to (9).

The following generalized Schmidt lemma holds.

Lemma 2 Let Hypothesis 1 be satisfied, $k=n$, and $\varphi_{i}, \psi_{i}(i=1, \ldots, n)$ be elements of $B_{1}^{*}$ and $B_{2}$ respectively such that

$$
\operatorname{det}\left[\varphi_{i}\left(\stackrel{\circ}{x}_{j}\right)\right] \neq 0, \quad \operatorname{det}\left[\stackrel{\circ}{x}_{i}^{*}\left(\psi_{j}\right)\right] \neq 0 \quad(i, j=1, \ldots, n) .
$$

Then:

(1) the operator $\underline{A}-\underline{A}_{1}$ is a Fredholm operator with zero index and equation (9) is uniquely and unconditionally solvable in $B_{1}$ for any $y \in B_{2}$;

(2) if $y \in B_{2}$ satisfies solvability conditions (8) of equation (4), then a solution $x$ of equation (9) is a solution of (4) such that

$$
\varphi_{i}(x)=0 \quad(i=1, \ldots, k) .
$$

Inversely, if $x$ is a solution of (9) such that conditions (12) are satisfied, then conditions (8) are satisfied for the right-hand side $y$ of equation (9) and $x$ is a solution of equation (4) with the same right-hand side $y$.

The proof of this lemma coincides, in fact, with the proof, which is given in $[6, \S 21]$, (see also [7, section 21.4]) for the particular case: $\varphi_{i}\left(\stackrel{\circ}{x}_{j}\right)=\stackrel{\circ}{x}_{i}^{*}\left(\psi_{j}\right)=\delta_{i j}$ (here $\delta_{i j}$ is the Kronecker symbol). A statement close to Lemma 2 includes also Lemma 4.8.23 in [10]. The lemma enables us to remove the spectrum point of equation (4) when some information about solutions of homogeneous equations (4) and (5) is available sufficient only for checking conditions (11).

Corollary 3 Under conditions of Lemma 2, equations

$$
\left(\underline{A}-\underline{A}_{1}\right) \hat{x}_{i}=\psi_{i}, \quad\left(\underline{A}^{*}-\underline{A}_{1}^{*}\right) \hat{x}_{i}^{*}=\varphi_{i}, i=1, \ldots, n
$$

are unconditionally and uniquely solvable and their solutions are such that $\operatorname{span}\left\{\hat{x}_{i}\right\}_{i=1}^{n}$ $=\operatorname{ker} \underline{A}, \varphi_{i}\left(\hat{x}_{j}\right)=-\delta_{i j} ; \operatorname{span}\left\{\hat{x}_{i}^{*}\right\}_{i=1}^{n}=\operatorname{ker} \underline{A}^{*}, \hat{x}_{i}^{*}\left(\psi_{j}\right)=-\delta_{i j}$.

Really, let $\hat{x}_{i}$ be a solution of first equation (13). By Lemma 2 this equation is unconditionally and uniquely solvable. Let us act on the equation by the functionals $\stackrel{\circ}{x}_{p}^{*}$ such that $\operatorname{ker} \underline{A}^{*}=\operatorname{span}\left\{\stackrel{\circ}{x}_{p}^{*}\right\}_{p=1}^{n}$ and obtain a linear algebraic system with respect to $\varphi_{j}\left(\hat{x}_{i}\right)$ for each fixed $i$ :

$$
-\sum_{j=1}^{n} \stackrel{\circ}{x}_{p}^{*}\left(\psi_{j}\right) \varphi_{j}\left(\hat{x}_{i}\right)=\stackrel{\circ}{x}_{p}^{*}\left(\psi_{i}\right) \quad(p=1, \ldots, n) .
$$

By second condition (11), this system is uniquely solvable and we can obtain by direct substituting that its solution is $\varphi_{j}\left(\hat{x}_{i}\right)=-\delta_{i j}$. After substituting this relation back into 
(13), we obtain that $\underline{A} \hat{x}_{i}=0$, that is, $\hat{x}_{i} \in \operatorname{ker} \underline{A}$. Finally, the linear independence of $\hat{x}_{i} \quad(i=1, \ldots, n)$ follows from the linear independence of the right-hand sides $\psi_{i}$ in (13). For the second equation (13), the proof is analogous.

This corollary allows to find eigen-solutions of original operators by solving a uniquely solvable perturbed equation.

Lemma 4 Let Hypothesis 1 be satisfied and $\varphi_{i}, \psi_{i}(i=1, \ldots, k \leq n)$ be elements of $B_{1}^{*}$ and $B_{2}$ respectively such that

$$
\begin{array}{rll}
\operatorname{det}\left[b_{i m}\right] \neq 0, & \operatorname{det}\left[b_{i m}^{*}\right] \neq 0 & (m, i=1, \ldots, k), \\
b_{i m}:=\varphi_{i}\left({ }^{\circ} x_{m}\right), & b_{i m}^{*}:=\stackrel{\circ}{x}_{m}^{*}\left(\psi_{i}\right) .
\end{array}
$$

Then:

(1) the operator $\underline{A}-\underline{A}_{1}$ is a Fredholm operator with zero index,

$$
\begin{aligned}
\operatorname{dim} \operatorname{ker}\left(\underline{A}-\underline{A}_{1}\right) & =\operatorname{dim} \operatorname{ker}\left(\underline{A}^{*}-\underline{A}_{1}^{*}\right)=n-k ; \\
\operatorname{ker}\left(\underline{A}-\underline{A}_{1}\right) & =\operatorname{span}\left\{\tilde{x}_{i}\right\}_{i=k+1}^{n} \subset \operatorname{ker} \underline{A}, \\
\operatorname{ker}\left(\underline{A}^{*}-\underline{A}_{1}^{*}\right) & =\operatorname{span}\left\{\tilde{x}_{i}^{*}\right\}_{i=k+1}^{n} \subset \operatorname{ker} \underline{A}^{*},
\end{aligned}
$$

where

$$
\begin{gathered}
\tilde{x}_{i}:=\stackrel{\circ}{x}_{i}-\sum_{j=1}^{k} \stackrel{\circ}{x}_{j} \sum_{p=1}^{k} b_{j p}^{-1} \varphi_{p}\left(\stackrel{\circ}{x}_{i}\right), \quad \tilde{x}_{i}^{*}:=\stackrel{\circ}{x}_{i}^{*}-\sum_{j=1}^{k} \stackrel{\circ}{x}_{j}^{*} \sum_{p=1}^{k} b_{j p}^{*-1} \stackrel{\circ}{x_{i}^{*}}\left(\psi_{p}\right), \\
i=k+1, \ldots, n .
\end{gathered}
$$

(2) If an element $y \in B_{2}$ satisfies solvability conditions (8) of equation (4), then equation (9) is also solvable for this $y$ and any its solution $x$ is a solution of (4) satisfying (12). Inversely, if equation (9) is solvable for an element $y \in B_{2}$ and its solution $x$ satisfies (12), then conditions (8) are also satisfied for $y$, and $x$ is a solution of equation (4) with the same right-hand side $y$.

Proof. The operator $\underline{A}-\underline{A}_{1}$ is a Fredholm operator since $\underline{A}_{1}$ is a finite-dimensional operator and $\underline{A}$ is a Fredholm operator. Let $\tilde{x}$ be a solution of the equation $\left(\underline{A}-\underline{A}_{1}\right) \tilde{x}=0$. Acting on this equation by the functionals $\stackrel{\circ}{x}_{j}^{*} \quad(j=1, \ldots, k)$, we obtain a linear algebraic system with respect to $\varphi_{i}\left(\tilde{x}_{i}\right)$,

$$
-\sum_{i=1}^{k} \stackrel{\circ}{x}_{j}^{*}\left(\psi_{i}\right) \varphi_{i}(\tilde{x})=0, \quad j=1, \ldots, k .
$$

By (14) it has only a trivial solution

$$
\varphi_{i}(\tilde{x})=0
$$

and, consequently, $\underline{A}_{1} \tilde{x}=0$ and $\tilde{x}$ is a solution of original homogeneous equation (4), that is

$$
\tilde{x}=\sum_{j=1}^{n} C_{j} \stackrel{\circ}{x}_{j} .
$$


Substituting (19) into (18) and taking into account the definition of $b_{m i}$, we obtain,

$$
\sum_{j=1}^{k} b_{i j} C_{j}+\sum_{j=k+1}^{n} C_{j} \varphi_{i}\left(\stackrel{\circ}{x}_{j}\right)=0, \quad(i=1, \ldots, k) .
$$

By (14) the matrix $b_{i j}(i, j=1, \ldots, k)$ is a regular matrix. Moving the second sum into the right-hand side, we solve the system with respect to $C_{j} \quad(j=1, \ldots, k)$ :

$$
C_{j}=-\sum_{p=1}^{k} b_{j p}^{-1} \sum_{i=k+1}^{n} C_{i} \varphi_{p}\left(\stackrel{\circ}{x_{i}}\right), \quad j=1, \ldots, k .
$$

Substituting this expressions into (19), we have

$$
\tilde{x}=\sum_{i=k+1}^{n} C_{i} \tilde{x}_{i}
$$

where $\tilde{x}_{i}$ are given in (16). and

We shall show that $\left(\underline{A}-\underline{A}_{1}\right) \tilde{x}_{i}=0$. Actually, $\underline{A} \tilde{x}_{i}=0$, as $\tilde{x}_{i}$ consists of $\stackrel{\circ}{x}_{j} \in \operatorname{ker} \underline{A}$,

$$
\begin{aligned}
\underline{A}_{1} \tilde{x}_{i} & =\sum_{q=1}^{k} \psi_{q} \varphi_{q}\left[\stackrel{\circ}{x}_{i}-\sum_{j=1}^{k} \stackrel{\circ}{x}_{j} \sum_{p=1}^{k} b_{j p}^{-1} \varphi_{p}\left(\stackrel{\circ}{x}_{i}\right)\right]= \\
& =\sum_{q=1}^{k} \psi_{q} \varphi_{q}\left(\stackrel{\circ}{x}_{i}\right)-\sum_{q=1}^{k} \psi_{q} \sum_{j=1}^{k} b_{q j} \sum_{p=1}^{k} b_{j p}^{-1} \varphi_{p}\left(\stackrel{\circ}{x}_{i}\right)=0 .
\end{aligned}
$$

Moreover, the elements $\tilde{x}_{i}(i=k+1, \ldots, n)$ are linearly independent since each of them is the sum of $\stackrel{\circ}{x}_{i}$ and the combination from $\stackrel{\circ}{x}_{j}(j=1, \ldots, k)$. Hence, there are exactly $n-k$ independent solutions of the equation $\left(\underline{A}-\underline{A}_{1}\right) x=0$.

By the same reasoning for the equation $\left(\underline{A}^{*}-\underline{A}_{1}^{*}\right) x^{*}=0$, we obtain second formula (16). The first part of the lemma is proved.

Let now $y$ satisfy (8), then it follows from (16) that

$$
\tilde{x}_{i}^{*}(y)=0 \quad(i=k+1, \ldots, n)
$$

and, hence, equation (9) is solvable with this right-hand side. As above, let us act on equation (9) by the functionals $\stackrel{\circ}{x}_{j}^{*}, j=1, \ldots, k$. Taking into account (8), we again obtain system (17) with respect to $\varphi_{i}(x)$. The system has only trivial solution (12) and, hence, $\underline{A}_{1} x=0$, that is, any solution of (9) is also solution of 4 .

Conversely, if the solvability conditions of equation (9) are satisfied and its solution satisfies (12), then $\underline{A}_{1} x=0$ and, hence, $x$ satisfies equation (4) with the same right-hand side $y$. Consequently, this right-hand side $y \in \mathcal{R}(\underline{A})$ and, hence, it satisfies (8). The second part of Lemma 4 is proved.

Lemma 4 enables to reduce the eigen-space dimension of equation (4). As in Lemma 2 , we are based on the rather poor information about eigen-solutions of homogeneous equations (4) and (5). This information is to be sufficient only to check conditions (14).

We have obvious corollaries from the proved Lemma. 
Corollary 5 Let the conditions of Lemma 4 be satisfied, then $\varphi_{p}\left(\tilde{x}_{i}\right)=0, \tilde{x}_{i}^{*}\left(\psi_{p}\right)=$ $0 \quad(p=1, \ldots, k, i=k+1, \ldots, n)$. If $\varphi_{p}\left(\stackrel{\circ}{x}_{i}\right)=0(p=1, \ldots, k, i=k+1, \ldots, n)$, then $\tilde{x}_{i}=\stackrel{\circ}{x}_{i} \quad(i=k+1, \ldots, n)$. Similarly, if $\stackrel{\circ}{x}_{i}^{*}\left(\psi_{p}\right)=0(p=1, \ldots, k, i=k+1, \ldots, n)$, then $\tilde{x}_{i}^{*}=\stackrel{\circ}{x}_{i}^{*}(i=k+1, \ldots, n)$.

Corollary 6 Let the conditions of Lemma 4 be satisfied. If $b_{i m}=-\delta_{i m}(i, m=1, \ldots, k)$, then

$$
\tilde{x}_{i}=\stackrel{\circ}{x}_{i}+\sum_{j=1}^{k} \stackrel{\circ}{x}_{j} \varphi_{j}\left(\stackrel{\circ}{x}_{i}\right) \quad(i=k+1, \ldots, n)
$$

Similarly, if $b_{i m}^{*}=-\delta_{i m}(i, m=1, \ldots, k)$, then

$$
\tilde{x}_{i}^{*}=\stackrel{\circ}{x}_{i}^{*}+\sum_{j=1}^{k} \stackrel{\circ}{x}_{j}^{*} \stackrel{o}{x}_{i}^{*}\left(\psi_{j}\right) \quad i=k+1, \ldots, n .
$$

An analogue of Corollary 3 is

Corollary 7 Let the conditions of Lemma 4 be satisfied. Then solutions $\hat{x}_{i}$ of the equations

$$
\left(\underline{A}-\underline{A}_{1}\right) \hat{x}_{i}=\psi_{i}, \quad i=1, \ldots, k
$$

are such that $\varphi_{i}\left(\hat{x}_{j}\right)=-\delta_{i j}$ and $\operatorname{ker} \underline{A}=\operatorname{span}\left\{\left\{\hat{x}_{i}\right\}_{i=1}^{k},\left\{\tilde{x}_{i}\right\}_{i=k+1}^{n}\right\}$, where $\left\{\tilde{x}_{i}\right\}_{i=k+1}^{n}$ are solutions of homogeneous equation (9).

Similarly, solutions $\hat{x}_{i}^{*}$ of the equations

$$
\left(\underline{A}^{*}-\underline{A}_{1}^{*}\right) \hat{x}_{i}^{*}=\varphi_{i}, \quad i=1, \ldots, k
$$

are such that $\hat{x}_{i}^{*}\left(\psi_{j}\right)=-\delta_{i j}$ and $\left.\operatorname{ker} \underline{A}^{*}=\operatorname{span}\left\{\left\{\hat{x}_{i}^{*}\right\}_{i=1}^{k},\left\{\tilde{x}_{i}^{*}\right\}_{i=k+1}^{n}\right\}\right\}$, where $\left\{\tilde{x}_{i}^{*}\right\}_{i=k+1}^{n}$ are solutions of the homogeneous equation (10).

Proof. Actually, let us consider, for example, equations (23). It follows from (16) that

$$
\begin{aligned}
\tilde{x}_{q}^{*}\left(\psi_{i}\right) & =\stackrel{\circ}{x}_{q}^{*}\left(\psi_{i}\right)-\sum_{j=1}^{k} \stackrel{\circ}{x}_{j}^{*}\left(\psi_{i}\right) \sum_{p=1}^{k} b_{j p}^{*-1} \stackrel{\circ}{x}_{q}^{*}\left(\psi_{p}\right)= \\
& =\stackrel{\circ}{x}_{q}^{*}\left(\psi_{i}\right)-\sum_{p=1}^{k} \delta_{i p} \stackrel{\circ}{x}_{q}^{*}\left(\psi_{p}\right)=0 \quad(i=1, \ldots, k, \quad q=k+1, \ldots, n)
\end{aligned}
$$

and hence equations (23) are solvable. It is taken into account here that $\stackrel{\circ}{x}_{j}^{*}\left(\psi_{i}\right)=b_{i j}^{*}$.

Let $\hat{x}_{i}$ be a solution of equation (23). Let us act on (23) by the functionals $\stackrel{\circ}{x}_{p}^{*}(p=$ $1, \ldots, k)$ and obtain a linear algebraic equation system with respect to $\varphi_{j}\left(\hat{x}_{i}\right)$ for every fixed $i$,

$$
-\sum_{j=1}^{k} \stackrel{\circ}{x}_{p}^{*}\left(\psi_{j}\right) \varphi_{j}\left(\hat{x}_{i}\right)=\stackrel{\circ}{x}_{p}^{*}\left(\psi_{i}\right) \quad(p=1, \ldots, k) .
$$

Because of second condition (14), this system is uniquely solvable and the direct substitution shows that $\varphi_{j}\left(\hat{x}_{i}\right)=-\delta_{i j}(i, j=1, \ldots, k)$. Substituting this relation into (23), we obtain that $\underline{A} \hat{x}_{i}=0$, that is, $\hat{x}_{i} \in \operatorname{ker} \underline{A}$. It follows from second condition (14) that $\psi_{i}$ are linearly independent. Then, by $(23)$, there is no linear combination of $\hat{x}_{i}$ belonging to $\operatorname{ker}\left(\underline{A}-\underline{A}_{1}\right)$. Hence, all elements of the set $\left\{\hat{x}_{i}\right\}_{i=1}^{k} \cup\left\{\tilde{x}_{i}\right\}_{i=k+1}^{n}$ are linear independent and each of this elements belongs to ker $\underline{A}$. Corollary 7 is proved for $\hat{x}_{i}$. The proof for $\hat{x}_{i}^{*}$ is similar. 


\section{Finite-dimensional perturbations for operator equa- tions of the second kind}

Let an operator $\underline{A}: B \rightarrow B$ be written in the form of a second-kind operator $\underline{A}=I-\lambda \underline{A}_{0}$. Equation (4) is transformed for this case into (6). We write its perturbed counterpart in the form

$$
\left[I-\lambda\left(\underline{A}_{0}+\underline{A}_{01}\right)\right] x=y, \quad \underline{A}_{01} x:=\sum_{i=1}^{k} \psi_{i} \varphi_{i}(x),
$$

where $\psi_{i}, \varphi_{i}$ are elements of $B$ and $B^{*}$ accordingly. Denote by $R(\lambda), R_{+}(\lambda)$ resolvents of the operators $\underline{A}_{0}$ and $\left(\underline{A}_{0}+\underline{A}_{01}\right)$, respectively, that is

$$
\begin{aligned}
\underline{R}(\lambda)\left(I-\lambda \underline{A}_{0}\right) & =I, & & \left(I-\lambda \underline{A}_{0}\right) \underline{R}(\lambda)=I, \\
\underline{R}_{+}(\lambda)\left[I-\lambda\left(\underline{A}_{0}+\underline{A}_{01}\right)\right] & =I, & & {\left[I-\lambda\left(\underline{A}_{0}+\underline{A}_{01}\right)\right] \underline{R}_{+}(\lambda)=I }
\end{aligned}
$$

at the $\lambda$-plane points, where these resolvents exist. To express $\underline{R}_{+}$through $\underline{R}$, let us act by the operator $\underline{R}(\lambda)$ on first equation (26) from the right and on the second equation from the left, and we get

$$
\underline{R}_{+}-\underline{R}=\lambda \sum_{j=1}^{k}\left(\underline{R}_{+} \psi_{j}\right)\left(\underline{R}^{*} \varphi_{j}\right), \quad \underline{R}_{+}-\underline{R}=\lambda \sum_{j=1}^{k}\left(\underline{R} \psi_{j}\right)\left(\underline{R}_{+}^{*} \varphi_{j}\right) .
$$

Acting now by the functionals $\varphi_{i}$ on second equation (27) we obtain a linear algebraic equation system to find $\underline{R}_{+}^{*} \varphi_{j}$ :

$$
\sum_{j=1}^{k}\left[\delta_{i j}-\lambda \varphi_{i}\left(\underline{R} \psi_{j}\right)\right] \underline{R}_{+}^{*} \varphi_{j}=\underline{R}^{*} \varphi_{i}
$$

Let

$$
W(\lambda)=\operatorname{det}\left[\delta_{i j}-\lambda \varphi_{i}\left(\underline{R} \psi_{j}\right)\right]
$$

be the number matrix determinant of this system (Weinstein-Aronszajn determinant) and $d_{i j}$ be its algebraic complements. Solving (28) and substituting the expression for $\underline{R}_{+}^{*} \varphi_{j}$ in (27), we obtain

$$
\underline{R}_{+}(\lambda)=\underline{R}\left[I+\frac{\lambda}{W(\lambda)} \sum_{i=1}^{k} \sum_{j=1}^{k} d_{i j} \psi_{j} \varphi_{i} \underline{R}\right] .
$$

Hence the singular points set of the resolvent operator to $\underline{A}_{0}+\underline{A}_{01}$ belongs to the union of the singular points of the resolvent operator to $\underline{A}_{0}$ and of the determinant $W(\lambda)$ zeros.

Using Lemma 2 or directly analysing representation (30) taking into account the resolvent operator expansion in the neighborhood of the pole [11], we get

Lemma 8 The singular point set of the resolvent operator $\underline{R}_{+}$belongs to the union of the resolvent operator $\underline{R}$ singular points and of determinant (29) zeros. Suppose $\lambda=\lambda_{0}$ is a finite order pole of the resolvent $\underline{R}(\lambda), k=n=\operatorname{dim} \operatorname{ker}\left(I-\lambda_{0} \underline{A}_{0}\right), \operatorname{span}\left\{\stackrel{\circ}{x}_{i}\right\}_{i=1}^{n}=$ $\operatorname{ker}\left(I-\lambda_{0} \underline{A}_{0}\right), \operatorname{span}\left\{\stackrel{\circ}{x}_{i}^{*}\right\}_{i=1}^{n}=\operatorname{ker}\left(I-\lambda_{0} \underline{A}_{0}^{*}\right)$, and conditions (11) are satisfied. Then $\lambda_{0}$ is a regular point of the resolvent $\underline{R}_{+}(\lambda)$. 
The following statement has been also proved (see [8], Theorem IV.6.2).

Lemma 9 The function $W(\lambda)$ from (29) is meromorphic in any domain of the $\lambda$-plane consisting of regular points of the resolvent $\underline{R}$ and of isolated eigen-values of the operator $\underline{A}_{0}$. For every $\lambda_{0}$ in such domain, the eigen-value algebraic multiplicity (the dimension of the subspace of eigen- and associated elements) of the operator $\underline{A}_{0}+\underline{A}_{01}$ is equal to the sum of the operator $\underline{A}_{0}$ eigen-value algebraic multiplicity and of multiplicity of the determinant $W$ zero at the point $\lambda_{0}$. The multiplicity of $W(\lambda)$ zero at a pole point of $W(\lambda)$ is taken as equal to its pole multiplicity with the minus sign.

Thus, if one can calculate or estimate zeros and poles of the determinant $W$, then one can analyse the singular points of the resolvent operator $\underline{R}_{+}(\lambda)$. Consider some cases when the determinant $W$ can be calculated explicitly.

Let us try to choose the elements $\varphi_{i}, \psi_{i}$ so that the operator $\underline{R}_{+}$is regular at the point $\lambda=\lambda_{0}$, where the operator $\underline{R}$ has a pole and on the other hand $\underline{R}_{+}$does not acquire additional (in comparison with $\underline{R}$ ) singular points in a finite part of the $\lambda$-plane.

Theorem 10 Let an operator $\underline{A}: B \rightarrow B, \quad \lambda_{0}$ be a simple pole of the resolvent $R(\lambda)$ for equation (6); $\operatorname{dim} \operatorname{ker}\left(I-\lambda_{0} \underline{A}_{0}\right)=n ; \operatorname{span}\left\{\stackrel{\circ}{x}_{i}\right\}_{i=1}^{n}=\operatorname{ker}\left(I-\lambda_{0} \underline{A}_{0}\right), \operatorname{span}\left\{\stackrel{\circ}{x}_{i}^{*}\right\}_{i=1}^{n}=$ $\operatorname{ker}\left(I-\lambda_{0} \underline{A}_{0}^{*}\right)$ and

$$
\varphi_{i}=\stackrel{\circ}{x}_{i}^{*}, \quad \stackrel{\circ}{x}_{i}^{*}\left(\psi_{j}\right)=-\delta_{i j} / \lambda_{0} \quad(i, j=1, \ldots, k)
$$

or

$$
\psi_{i}=\stackrel{\circ}{x}_{i}, \quad \varphi_{i}\left(\stackrel{\circ}{x}_{j}\right)=-\delta_{i j} / \lambda_{0} \quad(i, j=1, \ldots, k)
$$

and let $k=n$.

Then

(1). Singular points of the resolvent $\underline{R}_{+}(\lambda)$ for equation (24) coincide with singular points of the resolvent $\underline{R}(\lambda)$ for equation (6) and have the same algebraic multiplicities if these points are poles, excluding the point $\lambda=\lambda_{0}$, where the resolvent $\underline{R}_{+}(\lambda)$ is regular.

(2). If conditions (8) are satisfied, then a solution $x$ of equation (24) at $\lambda=\lambda_{0}$ is a solution of equation (6) and satisfies (12). Inversely, if $x$ is a solution of equation (24) at $\lambda=\lambda_{0}$ such that conditions (12) are satisfied, then conditions (8) are true for the righthand side $y$ of equation (24) and $x$ is a solution of equation (6) with the same right-hand side $y$.

(3). Under condition (31),

$$
\underline{R}(\lambda)=\underline{R}_{+}(\lambda)\left[I-\lambda \lambda_{0}\left(\lambda_{0}-\lambda\right)^{-1} \underline{A}_{01}\right], \quad \underline{R}_{+}(\lambda)=\underline{R}(\lambda)\left[I+\lambda \underline{A}_{01}\right] .
$$

Under condition (32),

$$
\underline{R}(\lambda)=\left[I-\lambda \lambda_{0}\left(\lambda_{0}-\lambda\right)^{-1} \underline{A}_{01}\right] \underline{R}_{+}(\lambda), \quad \underline{R}_{+}(\lambda)=\left[I+\lambda \underline{A}_{01}\right] \underline{R}(\lambda) .
$$

Proof. Suppose, for example, $\varphi_{i}=\stackrel{\circ}{x}_{i}^{*}$. Then $W(\lambda)=\operatorname{det}\left[\delta_{i j}-\lambda \stackrel{\circ}{x}_{i}^{*}\left(\underline{R} \psi_{j}\right)\right]$. Let $x_{i}^{*}:=$ $\stackrel{\circ}{x}_{i}^{*} \underline{R}(\lambda)=\underline{R}^{*}(\lambda) \stackrel{\circ}{x}_{i}^{*}$. By the definition of the resolvent, $\left(I-\lambda \underline{A}_{0}^{*}\right) x_{i}^{*}=\stackrel{\circ}{x}_{i}^{*}$ and if $\lambda$ is a resolvent regular point, then the solution of this equation is unique. Let us seek it in the 
form $x_{i}^{*}=C \stackrel{\circ}{x}_{i}^{*}$. Taking into account that $\underline{A}_{0}^{*} \stackrel{\circ}{x}_{i}^{*}=\stackrel{\circ}{x}_{i}^{*} / \lambda_{0}$, since $\stackrel{\circ}{x}_{i}^{*}$ is an eigen-solution of (7) at $\lambda=\lambda_{0}$, we obtain that $C=\lambda_{0} /\left(\lambda_{0}-\lambda\right)$. Hence,

$$
\stackrel{\circ}{x}_{i}^{*} \underline{R}=\lambda_{0}\left(\lambda_{0}-\lambda\right)^{-1} \stackrel{\circ}{x}_{i}^{*}
$$

and

$$
W(\lambda)=\operatorname{det}\left[\delta_{i j}-\lambda \lambda_{0}\left(\lambda_{0}-\lambda\right)^{-1}{ }^{\circ} x_{i}^{*}\left(\psi_{j}\right)\right]
$$

If $\stackrel{\circ}{x}_{i}^{*}\left(\psi_{j}\right)=-\delta_{i j} / \lambda_{0}$ according to (31), then $W(\lambda)=\lambda_{0}^{n}\left(\lambda_{0}-\lambda\right)^{-n}$ and hence $W(\lambda)$ has no zeros in a finite part of the $\lambda$-plane. Since $\lambda_{0}$ is a simple pole of $\underline{R}(\lambda)$, then (6) and, hence, (24) are Fredholm equations at $\lambda=\lambda_{0}$ (see, for example, [8]). Let us prove that $\operatorname{det}\left[\varphi_{i}\left(\stackrel{\circ}{x}_{j}\right)\right]=\operatorname{det}\left[\stackrel{\circ}{x}_{i}^{*}\left(\stackrel{\circ}{x}_{j}\right)\right] \neq 0$. Actually, otherwise there exist constants $C_{i} \quad(i=1, \ldots, n)$ such that $\stackrel{\circ}{x}_{j}^{*}(\stackrel{\circ}{x})=0$ for $\stackrel{\circ}{x}:=\sum_{i=1}^{n} C_{i} \stackrel{\circ}{x}$ for $j=1, \ldots, n$, that is, $\stackrel{\circ}{x} \in \mathcal{R}\left(I-\lambda_{0} \underline{A}_{0}\right)$, and then there exists an associated element $\tilde{x}:\left(I-\lambda_{0} \underline{A}_{0}\right) \tilde{x}=\stackrel{\circ}{x}$. This contradicts the fact that $\lambda_{0}$ is a resolvent simple pole [8].

Using Lemma 2, we obtain parts (1) and (2) under conditions (31).

Taking into account that $\underline{R}^{*} \varphi_{j}=\lambda_{0}\left(\lambda_{0}-\lambda\right)^{-1} \varphi_{j}$ owing to (35), we get first relation (33) from first relation (27). Let find $\hat{x}_{i}^{*}=\underline{R}_{+}^{*}{ }^{*}{ }_{i}^{*}$. According to the resolvent definition, $\left(I-\lambda \underline{A}_{0}^{*}-\lambda \underline{A}_{01}^{*}\right) \hat{x}_{i}^{*}=\stackrel{\circ}{x}_{i}^{*}$. Using (31) one can directly verify that $\hat{x}_{i}^{*}=\stackrel{\circ}{x}_{i}^{*}$ is the unique solution of this equation at any regular point $\lambda$ of the resolvent $\underline{R}_{+}$. That is $\underline{R}_{+}^{*}(\lambda) \varphi_{j}=\varphi_{j}$. Substituting this relation in second equation (27), we obtain second relation (33). This completes the proof of part (3) of the theorem under condition (31).

The theorem statements for the case (32) are proved similarly.

This theorem enables to remove a spectrum point possessing the information only about the eigen-solutions of the original equation or its conjugate equation. (Note that the classical Schmidt lemma requires us to know both of these eigen-sets for such spectral properties improvement.) Moreover, if a singular resolvent point $\lambda_{0}$ is removed by using this theorem and it is necessary to solve the equation at a regular point $\lambda$, then, according to the third part of the theorem, one can express a solution of the original equation for this value $\lambda$ in terms of the perturbed equation solution.

Note that statements similar to parts (1) and (2) of Theorem 10 for Hilbert spaces were presented in [12] and for Banach spaces in [9, §3].

Consider now an analogue of Lemma 4 for a second-kind equation, that is, a generalization of Theorem 10 for the case when the perturbing operator dimension is less then the eigen-subspace dimension for the operator $\underline{A}_{0}$ at $\lambda=\lambda_{0}$.

Theorem 11 Let all hypotheses of Theorem 10 be fulfilled excluding the condition $k=n$, which is replaced by the condition $k \leq n$. Then

(1). Singular points of the resolvent $\underline{R}_{+}(\lambda)$ for equation (24) coincide with singular points of the resolvent $\underline{R}(\lambda)$ for equation (6). The singular points have there the same algebraic multiplicities if these points are poles, excluding the point $\lambda=\lambda_{0}$, where the resolvent $\underline{R}_{+}(\lambda)$ has a simple pole and

$$
\begin{aligned}
\operatorname{dim} \operatorname{ker}\left[I-\lambda_{0}\left(\underline{A}_{0}+\underline{A}_{01}\right)\right] & =\operatorname{dim} \operatorname{ker}\left[I-\lambda_{0}\left(\underline{A}_{0}^{*}+\underline{A}_{01}^{*}\right)\right]=n-k, \\
\operatorname{ker}\left[I-\lambda_{0}\left(\underline{A}_{0}+\underline{A}_{01}\right)\right] & =\operatorname{span}\left\{\tilde{x}_{i}\right\}_{i=k+1}^{n} \subset \operatorname{ker}\left(I-\lambda_{0} \underline{A}_{0}\right), \\
\operatorname{ker}\left[I-\lambda_{0}\left(\underline{A}_{0}^{*}+\underline{A}_{01}^{*}\right)\right] & =\operatorname{span}\left\{\tilde{x}_{i}^{*}\right\}_{i=k+1}^{n} \subset \operatorname{ker}\left(I-\lambda_{0} \underline{A}_{0}^{*}\right) .
\end{aligned}
$$


For the case (31), $\tilde{x}_{i}^{*}$ are given by (22) and there exist $k$ elements $\stackrel{\circ}{x}_{i} \in \operatorname{ker}\left(I-\lambda_{0} \underline{A}_{0}\right)(i=$ $1, \ldots, k)$ such that $\operatorname{det}\left(b_{i m}\right) \neq 0, b_{i m}=\lambda_{0}{ }^{\circ}{ }_{i}^{*}\left(\stackrel{\circ}{x}_{m}\right)(i, m=1, \ldots, k)$, and $\tilde{x}_{i}$ are given by the first formula of (16). For the case (32), $\tilde{x}_{i}$ are given by (21) and there exist $k$ elements $\stackrel{\circ}{x}_{i}^{*} \in \operatorname{ker}\left(I-\lambda_{0} \underline{A}_{0}^{*}\right)(i=1, \ldots, k)$ such that $\operatorname{det}\left(b_{i m}^{*}\right) \neq 0, b_{i m}^{*}=\lambda_{0} \stackrel{\circ}{x}_{i}^{*}\left(\stackrel{\circ}{x}_{m}\right)(i, m=1, \ldots, k)$ and $\tilde{x}_{i}^{*}$ are given by the second formula of (16).

(2) If solvability conditions (8) of equation (6) are satisfied, then equation (24) is solvable at $\lambda=\lambda_{0}$ and any its solution $x$ is a solution of (6) and satisfies (12). Inversely, if equation (24) at $\lambda=\lambda_{0}$ is solvable and its solution $x$ satisfies (12), then conditions (8) are satisfied for the right-hand side $y$ of equation (24) and $x$ is a solution of equation (6) with this right-hand side $y$.

(3) Relationships (33) hold under condition (31) and relationships (34) hold under condition (32).

Proof. Repeating the same reasoning as by proving Theorem 10, we obtain that $W(\lambda)=\lambda_{0}^{k}\left(\lambda_{0}-\lambda\right)^{-k}$.

Moreover, in case (31), there exist $k$ linearly independent elements $\stackrel{\circ}{x}_{i} \in \operatorname{ker}(I-$ $\left.\lambda_{0} \underline{A}_{0}\right), i=1, \ldots, k$, such that $\operatorname{det}\left[\stackrel{\circ}{x}_{i}^{*}\left(\stackrel{\circ}{x}_{m}\right)\right] \neq 0(i, m=1, \ldots, k)$. Really, suppose this is not the case and consider the determinant $\operatorname{det}\left[a_{i m}\right]_{i, m=1}^{n}, a_{i m}=\stackrel{\circ}{x}_{i}^{*}\left(\stackrel{\circ}{x}_{m}\right)$. Then for any $k$ columns of the matrix there exists one column with a number $m_{1}$ such that $a_{i m_{1}}=\sum_{p=2}^{k} C_{p}^{\prime} a_{i m_{p}}, i=1, \ldots, k$. Subtracting the linear combination $\sum_{p=2}^{k} C_{p}^{\prime} a_{i m_{p}}, i=$ $1, \ldots, n$, from $m_{1}$-th column, we arrive at the same value of determinant but for a matrix that has zero elements at the $m_{1}$-th column, $a_{i m_{1}}^{\prime}=0, i=1, \ldots, k$. Repeating the process for another $k$ columns not including the $m_{1}$-th column, we arrive eventually at the determinant $\operatorname{det}\left[a_{i m}^{\prime}\right]_{i, m=1}^{n}= \pm \operatorname{det}\left[a_{i m}\right]_{i, m=1}^{n}$ of a matrix $a_{i m}^{\prime}$ such that $a_{i m_{p}}^{\prime}=0, i=1, \ldots, k, p=1, \ldots, n-k+1$. Then (see, e.g., [13, Section 1.6-5]) $\operatorname{det}\left[a_{i m}\right]_{i, m=1}^{n}= \pm \operatorname{det}\left[a_{i m_{p}}^{\prime}\right]_{i=1, \ldots, k ; p=n-k+1, \ldots, n} \operatorname{det}\left[a_{i m_{p}}^{\prime}\right]_{i=k+1, \ldots, n ; p=1, \ldots, n-k}=0$ since the first column in the first determinant of the right hand side equals to zero. This means, there exists a nonzero element $\stackrel{\circ}{x}:=\sum_{i=1}^{n} C_{i} \stackrel{\circ}{x}_{i}$, such that $\stackrel{\circ}{x}_{j}^{*}(\stackrel{\circ}{x})=0, j=1, \ldots, n$, that is, $\stackrel{\circ}{x} \in \mathcal{R}\left(I-\lambda_{0} \underline{A}_{0}\right)$, and then there exists an associated element $\tilde{x}:\left(I-\lambda_{0} \underline{A}_{0}\right) \tilde{x}=\stackrel{\circ}{x}$. This contradicts the fact that $\lambda_{0}$ is a resolvent simple pole [8].

One can prove similarly that in the case (32), there exist elements $\stackrel{\circ}{x}_{m}^{*} \in \operatorname{ker}(I-$ $\left.\lambda_{0} \underline{A}_{0}^{*}\right), m=1, \ldots, k$ such that $\operatorname{det}\left[\stackrel{\circ}{x}_{i}^{*}\left(\stackrel{\circ}{x}_{m}\right)\right] \neq 0(i, m=1, \ldots, k)$. Thus we get that the conditions of Lemma 4 are satisfied. Using Lemmas $2-4$ and Corollary 6 we obtain parts (1) and (2) of the theorem. Part (3) is proved in the same way as in Theorem 10.

Using Corollary 5, we obtained from Theorem 11 the obvious

Corollary 12 Let the hypotheses of Theorem 11 be satisfied.

(1) Suppose for the case (31), $\stackrel{\circ}{x}_{m} \in \operatorname{ker}\left(I-\lambda_{0} \underline{A}_{0}\right)(m=k+1, \ldots, n)$ are linearly independent elements such that $\stackrel{\circ}{x}_{i}^{*}\left(\stackrel{\circ}{x}_{m}\right)=0(i=1, \ldots, k, m=k+1, \ldots, n)$; then $\operatorname{ker}\left[I-\lambda_{0}\left(\underline{A}_{0}+\underline{A}_{01}\right)\right]=\operatorname{span}\left\{\stackrel{\circ}{x}_{m}\right\}_{m=k+1}^{n}$.

(2) Suppose for the case (32), $\stackrel{\circ}{x}_{m}^{*} \in \operatorname{ker}\left(I-\lambda_{0} \underline{A}_{0}^{*}\right)(m=k+1, \ldots, n)$ are linearly independent elements such that $\stackrel{\circ}{x}_{i}^{*}\left(\stackrel{\circ}{x}_{m}\right)=0(i=1, \ldots, k, m=k+1, \ldots, n)$; then $\operatorname{ker}\left[I-\lambda_{0}\left(\underline{A}_{0}^{*}+\underline{A}_{01}^{*}\right)\right]=\operatorname{span}\left\{\stackrel{\circ}{x}_{m}^{*}\right\}_{m=k+1}^{n}$.

Using Corollary 7 we obtain its analogue for second-kind equations. 
Corollary 13 Let the hypotheses of Theorem 11 be satisfied. Then solutions $\hat{x}_{j}$ of the equations

$$
\left[I-\lambda_{0}\left(\underline{A}_{0}+\underline{A}_{01}\right)\right] \hat{x}_{i}=\psi_{i}, \quad i=1, \ldots, k,
$$

are such that $\varphi_{i}\left(\hat{x}_{j}\right)=-\delta_{i j} / \lambda_{0}$ and $\operatorname{ker}\left(I-\lambda_{0} \underline{A}_{0}\right)=\operatorname{span}\left\{\left\{\hat{x}_{i}\right\}_{i=1}^{k},\left\{\tilde{x}_{i}\right\}_{i=k+1}^{n}\right\}$, where $\left\{\tilde{x}_{i}\right\}_{i=k+1}^{n}$ are solutions of homogeneous equation (36). Similarly, solutions $\hat{x}_{i}^{*}$ of the equations

$$
\left[I-\lambda_{0}\left(\underline{A}_{0}^{*}+\underline{A}_{01}^{*}\right)\right] \hat{x}_{i}^{*}=\varphi_{i}, \quad i=1, \ldots, k,
$$

are such that $\hat{x}_{j}^{*}\left(\psi_{j}\right)=-\delta_{i j} / \lambda_{0}$ and $\operatorname{ker}\left(I-\lambda_{0} \underline{A}_{0}^{*}\right)=\operatorname{span}\left\{\left\{\hat{x}_{i}^{*}\right\}_{i=1}^{k},\left\{\tilde{x}_{i}^{*}\right\}_{i=k+1}^{n}\right\}$, where $\left\{\tilde{x}_{i}^{*}\right\}_{i=k+1}^{n}$ are solutions of homogeneous equation (37).

Consider now the case when the operator $\underline{A}_{01}$ contains the terms satisfying (31) as well as the terms satisfying (32).

Theorem 14 Suppose the operator $\underline{A}_{0}: B \rightarrow B, \quad \lambda_{0}$ be a simple pole of the resolvent $\underline{R}(\lambda)$ for equation (6), $\operatorname{dim} \operatorname{ker}\left(I-\lambda_{0} \underline{A}_{0}\right)=n ; \varphi_{i} \in B^{*}, \psi_{i} \in B, i=1, \ldots, n, k=n, 0 \leq$ $t \leq n$

$$
\begin{gathered}
\varphi_{i}=\stackrel{\circ}{x}_{i}^{*} \quad(i=1, \ldots, t), \quad \psi_{j}=\stackrel{\circ}{x}_{j} \quad(j=t+1, \ldots, n) \\
\varphi_{i}\left(\psi_{j}\right)=-\delta_{i j} / \lambda_{0} \quad(i, j=1, \ldots, n),
\end{gathered}
$$

$\stackrel{\circ}{x}_{i}^{*}$ and $\stackrel{\circ}{x}_{j}$ are linear independent elements of $\operatorname{ker}\left(I-\lambda_{0} \underline{A}_{0}^{*}\right)$ and $\operatorname{ker}\left(I-\lambda_{0} \underline{A}_{0}\right)$ respectively.

Then statements (1) and (2) of Theorem 10 hold true and

$$
\begin{aligned}
\underline{R}(\lambda) & =\left[I-\lambda \lambda_{0}\left(\lambda_{0}-\lambda\right)^{-1} \underline{A}_{0 \varphi}\right] \underline{R}_{+}(\lambda)\left[I-\lambda \lambda_{0}\left(\lambda_{0}-\lambda\right)^{-1} \underline{A}_{0 \psi}\right] \\
\underline{R}_{+}(\lambda) & =\left[I+\lambda \underline{A}_{0 \varphi}\right] \underline{R}(\lambda)\left[I+\lambda \underline{A}_{0 \psi}\right]
\end{aligned}
$$

where

$$
\underline{A}_{0 \psi}:=\sum_{j=1}^{t} \psi_{j} \stackrel{\circ}{x}_{j}^{*}, \quad \underline{A}_{0 \varphi}:=\sum_{j=t+1}^{n} \stackrel{\circ}{x}_{j} \varphi_{j}, \quad \underline{A}_{01}=\underline{A}_{0 \psi}+\underline{A}_{0 \varphi} .
$$

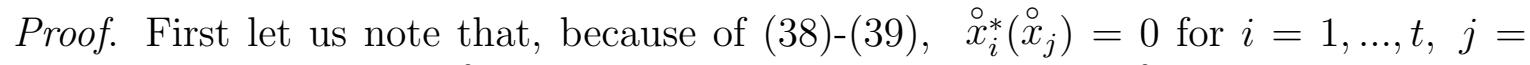
$t+1, \ldots, n$, and the elements $\stackrel{\circ}{x}_{i}^{*}(i=1, \ldots, t)$ as well as the elements $\stackrel{\circ}{x}_{j}(j=t+1, \ldots, n)$ are linearly independent. Consider the equation $\left[I-\lambda_{0}\left(\underline{A}_{0}+\underline{A}_{0 \psi}\right)\right] x=y$ for which Theorem 11 with condition (31) and part 1 of Corollary 12 hold true. Hence, $\left.\operatorname{ker}\left(I-\lambda_{0} \underline{\tilde{A}}_{0}\right)\right)=$ $\operatorname{span}\left\{\stackrel{\circ}{x}_{j}\right\}_{j=k+1}^{n}$ for the operator $\underline{\tilde{A}}_{0}:=\underline{A}_{0}+\underline{A}_{0 \psi}$. Applying Theorem 10 to the equation $\left[I-\lambda_{0} \underline{\tilde{A}}_{0}\right] x=y$, we conclude the proof.

Using Corollary 3 we get its analogue for a second-kind operator.

Corollary 15 Let the hypotheses of Theorem 10 or 14 be satisfied, then the equations

$$
\left[I-\lambda_{0}\left(\underline{A}_{0}+\underline{A}_{01}\right)\right] \hat{x}_{i}=\psi_{i}, \quad\left[I-\lambda_{0}\left(\underline{A}_{0}^{*}+\underline{A}_{01}^{*}\right)\right] \hat{x}_{i}^{*}=\varphi_{i}, \quad i=1, \ldots, n
$$

are unconditionally and uniquely solvable and their solutions are such that

$$
\begin{array}{ll}
\operatorname{span}\left\{\hat{x}_{i}\right\}_{i=1}^{n}=\operatorname{ker}\left(I-\lambda_{0} \underline{A}_{0}\right), & \varphi_{i}\left(\hat{x}_{j}\right)=-\delta_{i j} / \lambda_{0}, \text { and } \\
\operatorname{span}\left\{\hat{x}_{i}^{*}\right\}_{i=1}^{n}=\operatorname{ker}\left(I-\lambda_{0} \underline{A}_{0}^{*}\right), & \hat{x}_{i}^{*}\left(\psi_{j}\right)=-\delta_{i j} / \lambda_{0} .
\end{array}
$$




\section{On calculation of undetermined constants in the equation right-hand side}

Consider now Fredholm equation (4), where $\underline{A}: B_{1} \rightarrow B_{2}, \operatorname{dim} \operatorname{ker} \underline{A}=\operatorname{dim} \operatorname{ker} \underline{A}^{*}=n$ and $y=y_{0}+\sum_{j=1}^{n} C_{j} y_{j}, y_{j} \in B_{2}(j=0, \ldots, n)$. One should choose the constants $C_{j}$ such that solvability conditions (8) of equation (4), will be satisfied, that is,

$$
\stackrel{\circ}{x}_{i}^{*}\left(y_{0}+\sum_{j=1}^{n} C_{j} y_{j}\right)=0, \quad i=1, \ldots, n, \quad \operatorname{span}\left\{\stackrel{\leftrightarrow}{x}_{i}^{*}\right\}_{i=1}^{n}=\operatorname{ker} \underline{A},
$$

and also find one of solutions to equation (4).

It is obvious that this problem is solvable in the general case only if

$$
\operatorname{det}\left(\stackrel{\circ}{x}_{i}^{*} y_{j}\right) \neq 0 \quad(i, j=1, \ldots, n) .
$$

Suppose this holds true.

If the functionals $\stackrel{\circ}{x}_{i}^{*}$ are known, then one can find $C_{j}$ from (42) and then, using Lemma 2 (or Theorems 10, 14 if $\underline{A}: B \rightarrow B$ is a second-kind operator), one can perturb the equation and obtain the solution by solving corresponding unconditional and uniquely solvable equation (9).

If the functionals $\stackrel{\circ}{x}_{i}^{*}$ are unknown, then there are at least two ways forward. Firstly, one can find $\stackrel{\circ}{x}_{i}^{*}$ by Corollary 3 from the second group of perturbed equations (13) (or by Corollary 15 from the second group of equations (41) if $\underline{A}$ is the second-kind operator) and then do as above.

Secondly, one can perturb equation (4) by Lemma 2 (or by Theorems 10, 14 if $\underline{A}$ is a second-kind operator) and find its solutions $x_{j}$ with the right-hand sides $y_{j} \quad(j=0, \ldots, n)$ respectively. Then one can demand that the solution

$$
x=x_{0}+\sum_{j=1}^{n} C_{j} x_{j}
$$

satisfies condition (12) according to the second part of Lemma 2 (Theorems 10, 14). This leads to a linear algebraic equation system with respect to $C_{j}$ :

$$
\sum_{j=1}^{n} C_{j} \varphi_{i}\left(x_{j}\right)=-\varphi_{i}\left(x_{0}\right)(i=1, \ldots, n) .
$$

Let us show that $\operatorname{det}\left[\varphi_{i}\left(x_{j}\right)\right] \neq 0$ under condition (43). Really, otherwise non-zero constants $C_{j}^{0}$ can be found such that

$$
\varphi_{i}\left(\sum_{j=1}^{n} C_{j}^{0} x_{j}\right)=0, \quad i=1, \ldots, n .
$$

According to the second part of Lemma 2 (Theorems 10, 14), this means that

$$
\sum_{j=1}^{n} C_{j}^{0} x_{i}^{*}\left(y_{j}\right)=0, \quad i=1, \ldots, n
$$

but it is in contradiction to (43).

Thus one can solve the problem also by this second way. 


\section{Applications to boundary integral equations}

We shall illustrate now on a BIE of elasticity how one can apply the above results. We consider BIE (1) from the introduction. If $S \in C^{1, \alpha}$, then (see $[3,4,5]$ ) the operator $I+\underline{W}$ satisfies Hypothesis 1 for $n=6, \operatorname{ker}(I+\underline{W})=\operatorname{span}\left\{\stackrel{\circ}{u}^{(m)}\right\}_{m=1}^{6}$ (the eigen-solutions $\stackrel{\circ}{u}_{i}^{(m)}$ are given in $\left.(2)\right), B_{1}=B_{2}=C^{0, \beta}(S), 0<\beta<\alpha$. For a nonsmooth surface $S$, the Hypothesis will be satisfied in some weighted Hölder spaces $B_{1}=B_{2}$ with the same $\operatorname{ker}(I+\underline{W})$, see [5].

Let us denote by $|S|$ the area, by $\eta^{c}$ the center of inertia, and by $J$ the central moment if inertia (the first invariant of the inertia tensor) for the surface $S$, that is,

$$
|S|:=\int_{S} d S, \quad \eta_{i}^{c}:=\frac{1}{|S|} \int_{S} \eta_{i} d S, \quad J:=\int_{S}\left(\eta_{i}-\eta_{i}^{c}\right)\left(\eta_{i}-\eta_{i}^{c}\right) d S(\eta)
$$

Suppose firstly, the coordinate axes $\eta_{i}$ are parallel to the principal axes of the inertia tensor for the surface $S$, that is,

$$
\int_{S}\left(\eta_{i}-\eta_{i}^{c}\right)\left(\eta_{j}-\eta_{j}^{c}\right) d S(\eta)=0, i \neq j
$$

We write the perturbed equation corresponding to (1) in the form

$$
\begin{gathered}
u_{i}(\xi)-\lambda\left\{\left[\underline{W}_{i j}+\underline{K}_{i j}^{(31)}\right] u_{j}\right\}(\xi)=\Phi_{i}(\xi), \\
{\left[\underline{K}_{i j}^{(31)} u_{j}\right](\xi):=\sum_{m=1}^{3}\left[\stackrel{\circ}{u}_{i}^{(m)}\left(\xi-\eta^{c}\right) \int_{S} \phi_{j}^{(m)}\left(\eta-\eta^{c}\right) u_{j}(\eta) d S(\eta)\right.} \\
\\
\left.+\stackrel{\circ}{u}_{i}^{(3+m)}\left(\xi-\eta^{c}\right) \int_{S} \phi_{j}^{(3+m)}\left(\eta-\eta^{c}\right) u_{j}(\eta) d S(\eta)\right] .
\end{gathered}
$$

The functions $\phi_{j}^{(m)}$ in (44) are chosen in the form

$$
\phi_{j}^{(m)}(\xi)=\frac{1}{|S|} \stackrel{\circ}{u}_{j}^{(m)}(\xi)=\frac{\delta_{m j}}{|S|}, \phi_{j}^{(3+m)}(\xi)=\frac{1}{2 J} \stackrel{\circ}{u}_{j}^{(3+m)}(\xi)=\frac{\epsilon_{j p m} \xi_{p}}{2 J}, \quad m=1, \ldots, 3 .
$$

Then it is easy to check, that the perturbing operator $\underline{K}^{(31)}$ satisfies Theorem 10 (with condition (32)) for $k=n=6, \lambda_{0}=-1$,

$$
\begin{gathered}
\stackrel{\circ}{x} \text { m,i }_{(\xi)=\delta_{i m}, \quad} \stackrel{\stackrel{\circ}{x}_{m+3, i}(\xi)=\epsilon_{i j m}\left(\xi_{j}-\eta_{j}^{c}\right),}{ } \\
\varphi_{m}(u)=\frac{1}{|S|} \int_{S} u_{m}(\eta) d S(\eta), \quad \varphi_{m+3}(u)=\frac{1}{2 J} \int_{S} \epsilon_{j p m}\left(\eta_{p}-\eta_{p}^{c}\right) u_{j}(\eta) d S(\eta), \quad m=1, \ldots 3 .
\end{gathered}
$$

Consequently, BIE (44) is uniquely and unconditionally solvable at $\lambda_{0}=-1$. Its solution $u_{i}$ coincides with one of the solutions of BIE (1) such that

$$
\int_{S} u_{i}(\eta) d S=0, \int_{S} \epsilon_{i j m} u_{i}(\eta)\left(\eta_{j}-\eta_{j}^{c}\right) d S=0, \quad i, j, m=1, \ldots, 3
$$


if the total force and the moment of the applied tractions equal zero (what implies the solvability conditions (3) for BIE (1) are satisfied). Moreover, the resolvent of the perturbed operator has the same singular points as the resolvent of the original operator excluding the point $\lambda=-1$. It means that the resolvent is now regular in the closed circle $|\lambda| \leq 1$. Hence, perturbed equation (44) can be solved at $\lambda=-1$, e.g., by the method of simple iterations, that is, by expansion of the resolvent in the convergent Neumann series.

After using the property $\epsilon_{i j k} \epsilon_{m l k}=\delta_{i m} \delta_{j l}-\delta_{i l} \delta_{j m}$, we can represent the perturbing operator in a simpler form

$$
\begin{aligned}
{\left[\underline{K}_{i j}^{(31)} u_{j}\right](\xi)=\int_{S}\left\{\frac{1}{|S|} u_{i}(\eta)+\right.} & \frac{1}{2 J}\left[\left(\xi_{j}-\eta_{j}^{c}\right)\left(\eta_{j}-\eta_{j}^{c}\right) u_{i}(\eta)\right. \\
& \left.\left.-\left(\xi_{j}-\eta_{j}^{c}\right)\left(\eta_{i}-\eta_{i}^{c}\right) u_{j}(\eta)\right]\right\} d S(\eta) .
\end{aligned}
$$

One can remark that the presentation (45) is true also in arbitrary cartesian coordinate system (not only associated with the principal axes of the inertia tensor), since the righthand side of (45) is a linear combination of vectors, which coefficients are scalar products of vectors.

This perturbation technique can be used also for other BIEs. For example, an application of perturbation operators to BIE of harmonic functions is presented in $[9,14]$, and to BIE of plane elastic problems in [15]. An implementation to BIE, obtained by the indirect approach for elastic plate reinforced by boundary curvilinear elastic bars, was described in [16]. Determination of unknown constants in the BIE right-hand side by methods of Section 4 . was used in $[15,16]$.

Several results of this paper were announced in [17].

\section{References}

1. Muskhelishvili, N. I. Some Basic Problems of Mathematical Theory of Elasticity. Groningen, Noordhoff, 1953.

2. Brebbia, C. A., Telles, J. C. F. \& Wrobel L. C. Boundary Element Techniques: Theory and Application in Engineering. Springer-Verlag, Berlin-Heidelberg-New-York-Tokyo, 1984.

3. Kupradze, V.D., Gegelia, T.G., Basheleishvili, M.O., Burchuladze, T.V. ThreeDimensional Problems of the Mathematical Theory of Elasticity and Thermoelasticity. Nauka, Moscow, 1976.

4. Parton, V. Z. \& Perlin, P. I. Methods of Mathematical Theory of Elasticity. Nauka, Moscow, 1981.

5. Maz'ya V.G. Boundary Integral Equations. In: Analysis IV. Linear and Boundary Integral Equations, ed. V.G.Maz'ya \& S.M.Nikol'skii, Springer-Verlag, BerlinHeidelberg, etc., 1991, pp. 127-233.

6. Vainberg, M. M. \& Trenogin V. A. Theory of Branching of Solutions of Non-Linear Equations. Noordhoff International Pub, Leyden, 1974. 
7. Trenogin, V. A. Functional analysis. Nauka, Moscow, 1980 (in Russian).

8. Kato, T. Perturbation Theory for Linear Operators. Springer-Verlag, New-York, 1966.

9. Mikhailov, S. E. On an integral equation of some boundary value problems for harmonic functions in plane multiply connected domains with nonregular boundary, Matematicheskii Sbornik, 1983, 121, 533-544. (Engl. Translation: Mathematics of the USSR. - Sbornik, 1984, 49, 525-536.)

10. Hackbusch, W. Integral Equations: Theory and Numerical Treatment. Birkhäuser, Basel, 1995.

11. Krachkovsky, S. N. On expending domain of singularity for operator $T_{\lambda}=E-\lambda A$, Dokl. AN SSSR, 1954, 94, 1101-1104 (in Russian).

12. Lazarev, M. I. \& Perlin, P. I. On one way of construction of approximate solution to operator equation on spectrum, Izv. VYZov. Ser. Math., 1980, No. 3, 19-21 (in Russian).

13. Korn, G.A. \& Korn, T.M. Mathematical Handbook. McGrow-Hill Book Company, New-York etc., 1968.

14. Mikhailov, S.E. Solution of problems on the anti-plane deformation of elastic bodies with corner points by the method of integral equations, J. of Appl. Math. and Mech. (PMM), 1983, 47, 783-788.

15. Mikhailov, S.E. Spectral properties and solution methods for some integral equations of elasticity for plane non-simply-connected bodies with corner points, under forces specified on the boundary, Mech. of Solids (Izv.AN SSSR.MTT), 1989 24(5), 53-63.

16. Mikhailov, S.E. Certain boundary integral equations of the plane problem of elasticity theory for nonsingly connected bodies with one-dimensional elastic borders and angle points, Mech. of Solids (Izv. AN SSSR. MTT). 1992, 27(1), 36-47.

17. Mikhailov, S.E. Application of finite-dimensional perturbation technique to boundary integral equations. In: Boundary Elements XX, Conference proceedings, 19-21 August 1998, Orlando, Florida, USA, ed. A.Kassab, M.Chopra \& C.A.Brebbia, Computational Mechanics Publ., Southampton-Boston 1998, pp. 321-332. 